\title{
Circuit Lower Bounds for Nondeterministic Quasi-Polytime: An Easy Witness Lemma for NP and NQP
}

\author{
Cody Murray \\ MIT \\ USA
}

\begin{abstract}
We prove that if every problem in NP has $n^{k}$-size circuits for a fixed constant $k$, then for every NP-verifier and every yes-instance $x$ of length $n$ for that verifier, the verifier's search space has an $n^{O}\left(k^{3}\right)$ size witness circuit: a witness for $x$ that can be encoded with a circuit of only $n^{O\left(k^{3}\right)}$ size. An analogous statement is proved for nondeterministic quasi-polynomial time, i.e., NQP $=\mathrm{NTIME}\left[n^{\log { }^{(1)} n}\right]$. This significantly extends the Easy Witness Lemma of Impagliazzo, Kabanets, and Wigderson [JCSS'02] which only held for larger nondeterministic classes such as NEXP.

As a consequence, the connections between circuit-analysis algorithms and circuit lower bounds can be considerably sharpened: algorithms for approximately counting satisfying assignments to given circuits which improve over exhaustive search can imply circuit lower bounds for functions in NQP, or even NP. To illustrate, applying known algorithms for satisfiability of ACC $\circ$ THR circuits [R. Williams, STOC 2014] we conclude that for every fixed $k$, NQP does not have $n^{\log ^{k} n}$-size ACC $\circ$ THR circuits.
\end{abstract}

\section{CCS CONCEPTS}

- Theory of computation $\rightarrow$ Complexity classes; Circuit complexity; Pseudorandomness and derandomization;

\section{KEYWORDS}

circuit complexity, lower bounds, nondeterminism, Merlin-Arthur, ACC, satisfiability, pseudorandomness

ACM Reference Format:

Cody Murray and Ryan Williams. 2018. Circuit Lower Bounds for Nondeterministic Quasi-Polytime: An Easy Witness Lemma for NP and NQP. In Proceedings of 50th Annual ACM SIGACT Symposium on the Theory of Computing (STOC'18). ACM, New York, NY, USA, 12 pages. https://doi.org/ $10.1145 / 3188745.3188910$

\section{INTRODUCTION}

Proving non-uniform circuit lower bounds for functions in nondeterministic complexity classes such as NP or NEXP $=\operatorname{NTIME}\left[2^{n^{O(1)}}\right]$

*Supported by NSF CAREER award CCF-1552651.

Permission to make digital or hard copies of all or part of this work for personal or classroom use is granted without fee provided that copies are not made or distributed for profit or commercial advantage and that copies bear this notice and the full citation on the first page. Copyrights for components of this work owned by others than the author(s) must be honored. Abstracting with credit is permitted. To copy otherwise, or republish, to post on servers or to redistribute to lists, requires prior specific permission and/or a fee. Request permissions from permissions@acm.org.

STOC'18, June 25-29, 2018, Los Angeles, CA, USA

(C) 2018 Copyright held by the owner/author(s). Publication rights licensed to the Association for Computing Machinery.

ACM ISBN 978-1-4503-5559-9/18/06 \$15.00

https://doi.org/10.1145/3188745.3188910

\author{
Ryan Williams* \\ MIT \\ USA
}

is a well-known grand challenge. A natural approach is to assume that a given complexity class has small circuits, and to derive unlikely consequences, eventually absurd enough to derive a contradiction with other results. Over the years, interesting consequences have been derived from assuming (for example) NP has $O(n)$-size circuits, or NEXP has polynomial-size circuits; for a sample, see $[7,11,12,14-17,28]$.

One of the most striking results in this line of work is the Easy Witness Lemma of Impagliazzo, Kabanets, and Wigderson [12]. Informally, they show that if NEXP has small circuits, then every yes-instance of every NEXP problem has a highly compressible "easy" witness:

Lemma 1.1 (EAsy Witness Lemma [12] (Informal)). If NTIME $\left[2^{n}\right]$ has circuits of size $s(n)$, then there is a $k$ such that every yes-instance for every verifier for every problem in NTIME $\left[2^{n}\right]$ has a witness string $w$ (of $O\left(2^{n}\right)$ length) which can be encoded by a circuit of size at most $s\left(s\left(n^{k}\right)^{k}\right)^{k}$. In particular, viewing $w$ as the truth table of a function $f_{w}:\{0,1\}^{n+O(1)} \rightarrow\{0,1\}$, this $f_{w}$ has $s\left(s\left(n^{k}\right)^{k}\right)^{k}$-size circuits.

For $s(n)$ being polynomial or quasipolynomial (in other words, $\left.s(n)=2^{\log ^{O(1)} n}\right)$, Lemma 1.1 says that small circuits for NEXP imply small circuits encoding solutions to NEXP problems. Informally, we say the consequence of Lemma 1.1 means NTIME $\left[2^{n}\right]$ has small witness circuits. Note an NEXP search problem has $2^{2^{\text {poly(n)}}}$ possible solutions; having small witness circuits means that the existence of some witness in this huge space implies an "easy" witness with a short description, in a much smaller search space. Furthemore, if NTIME $\left[2^{n}\right]$ has poly-size witness circuits, then NEXP = EXP, by enumerating over all $2^{\text {poly }(n)}$ small circuits and checking if any encode a witness for the given verifier. Thus Lemma 1.1 shows NEXP $\subset$ P/poly implies NEXP $=$ EXP. Impagliazzo, Kabanets, and Wigderson found many other applications, including a surprising equivalence between NEXP $\not \subset \mathrm{P} /$ poly and non-trivial derandomizations.

Application: NEXP Lower Bounds. The second author [26, 27] used the Easy Witness Lemma (along with other ideas) to prove NEXP does not have $n^{\log { }^{(1)}} n$-size ACC circuits (with the polylog factors tightened in [6]), and NEXP does not have $n^{\log { }^{O(1)}} n_{\text {-size ACC } 0 \text { THR }}$ circuits (ACC composed with a layer of linear threshold gates at the bottom). These results were proved by designing faster-than- $2^{n}$ algorithms for the SAT problem for ACC circuits, and using the algorithms in an involved argument with the Easy Witness Lemma which (eventually) applies the nondeterministic time hierarchy [25]. In general, improving the time complexity of CIRCUIT SAT (or even 
estimating the fraction of satisfying assignments to within a small constant, which is widely believed to be in $\mathrm{P}$ ) for a "typical" complexity class $C$ (such as ACC, $\mathrm{TC}^{0}, \mathrm{NC}^{1}, \mathrm{NC}, \mathrm{P} /$ poly) from $2^{n} \cdot s^{O(1)}$ time to (for example) $2^{n} \cdot s^{O(1)} / n^{\text {poly }(\log n)}$ time would imply NEXP does not have $C$-circuits of $n^{\log n}$ size [4,21, 25]. This algorithmic approach to proving circuit lower bounds via weak circuit satisfiability algorithms has led to some optimism that lower bounds like NEXP $\not \subset \mathrm{P} /$ poly may be approachable, and provable.

The Quest for Lower-Complexity Functions. While this program for proving lower bounds has seen some successes, a major deficiency is that it can only yield circuit lower bounds for functions in NTIME $\left[2^{n^{\varepsilon}}\right]$ and higher, for $\varepsilon>0$. As NEXP is presumably an enormous class, results such as NEXP $\not \subset$ ACC are far less interesting than proving that a more explicit function, such as SAT, is not in ACC. A key bottleneck in the arguments is the Easy Witness Lemma: Lemma 1.1 is crucial in the connection between SAT algorithms and lower bounds, but its proof only works for nondeterministic exponential-time classes. ${ }^{1}$ Given an Easy Witness Lemma for NP (i.e., if NP has $n^{k}$-size circuits, then every yesinstance of every NP problem has $n^{f(k)}$-size witness circuits), or for NQP $=$ NTIME $\left[n^{\log ^{O(1)} n}\right]$, circuit lower bounds for these smaller classes could be obtained as well, provided that the necessary (weak) Circuit SAT algorithms are fast enough. ${ }^{2}$ Prior work tried to avoid this problem by working with the larger classes $\mathrm{QP}^{\mathrm{NP}}$ [27] or $\mathrm{P}^{\mathrm{NP}}$ [11], but these are less natural and less obviously weaker (it is open whether NEXP $=\mathrm{P}^{\mathrm{NP}}$ !).

\subsection{Easy Witness Lemmas for NP and NQP and Applications.}

In this paper, we prove an easy witness lemma that works for both NP and NQP. These results readily imply (using the existing framework) stronger circuit lower bounds: for example, NQP does not have ACC $\circ$ THR circuits of $n^{\log ^{k} n}$ size, for all fixed $k$.

LEMMA 1.2 (EASY WitNESSES FOR NP). There is a $c \geq 1$ such that for all $k \geq 1$, if NP $\subset \mathrm{SIZE}\left[n^{k}\right]$ then every $L \in \mathrm{NP}$ has witness circuits of size at most $n^{c k^{3}}$.

LEMMA 1.3 (EASY WitnesSES FOR NQP). There is a $c \geq 1$ such that for all $k \geq 1$, if NQP $\subset \mathrm{SIZE}\left[2^{\log ^{k} n}\right]$ then every $L \in \mathrm{NQP}$ has witness circuits of size $2^{c \log ^{k^{3}} n}$.

The full formal statement encapsulating both lemmas can be found in Lemma 4.1. Our primary motivation for proving these lemmas is to improve the known generic connections between circuit-SAT algorithms and circuit lower bounds. We only need the underlying class $C$ of Boolean circuits to satisfy a few simple properties. Informally, a circuit class $C$ is evaluatable if given a

\footnotetext{
${ }^{1}$ There are simple easy witness lemmas for deterministic classes such as P and EXP, as one can see from $[11,17]$. But it is not known how to extend the connection from SAT algorithms to circuit lower bounds to prove non-uniform lower bounds for functions in deterministic classes such as P and EXP; a crucial aspect of those arguments is that the non-uniform circuit is nondeterministically guessed in the eventual proof-bycontradiction.

${ }^{2}$ It's worth noting that proving NP doesn't have circuits of size $n^{k}$ would have other major implications, such as NEXP $\not \subset \mathrm{P}$ /poly and non-trivial derandomizations of MA [12].
}

description of a $C$ from $C$ and an assignment $x$ to some of the inputs, it is easy to evaluate $C(x) ; C$ is closed under negation if it easy to compute the negation of a given circuit from $C ; C$ is closed under conjunctions if it is easy to take a collection of $C$-circuits, each with $n$ inputs, and output one $n$-input $C$-circuit equivalent to the conjunction of the given circuits. Classes with all three properties are called typical. (Definitions are in the Preliminaries.)

For any circuit class $C$, let $C$-SAT be the satisfiability problem for circuits from $C$. We state our results in terms of a presumably much easier problem, the GAP $C$ UNSAT problem, in which we are promised that the given $C$-circuit on $n$ inputs is either unsatisfiable, or has at least $2^{n} / 4$ satisfying assignments. Unlike $C$-SAT, the GAP $C$ UnSAT problem is trivially solvable with randomness. (In prior work [21] the problem is called DeRANDOMIZE- $C$; another version is called CAPP [12].) We say a nondeterministic algorithm solves GAP $C$ UnSAT if:

- it outputs yes, no, or don't know on every computation path,

- if its input circuit has at least $2^{n} / 4$ satisfying assignments, then it outputs no on some path,

- if its input circuit is unsatisfiable, then it outputs yes on some path, and

- for every input circuit, it never outputs yes on some path and no on another path.

It is widely believed that GAP $C$ UNSAT is solvable in deterministic polynomial time; this would follow from the existence of pseudorandom generators sufficient for $\mathrm{P}=\mathrm{BPP}$. With this notation, our new algorithms-to-lower bounds connections can be stated:

Theorem 1.4 (NP Lower Bounds From Faster Circuit-SAT). Let $C$ be typical, and let $\varepsilon \in(0,1)$. Suppose GAP $C$ UnSAT on $n$ input, $2^{\varepsilon n}$-size circuits is solvable in nondeterministic $O\left(2^{(1-\varepsilon) n}\right)$ time. Then there is a $c \geq 1$ such that for all $k$, NTIME $\left[n^{c k^{4} / \varepsilon}\right]$ does not have $n^{k}$-size $C$-circuits. ${ }^{3}$

Theorem 1.5 (NQP Lower Bounds From Faster Circuit-SAT). Let $C$ be typical, and let $\varepsilon \in(0,1)$. Suppose GAP $C$ Unsat on $n$ input, $2^{n^{\varepsilon}}$-size circuits is in nondeterministic $O\left(2^{n-n^{\varepsilon}}\right)$ time. Then

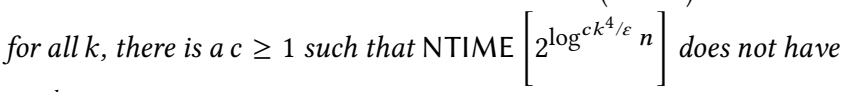
$2^{\log ^{k} n}$-size $C$-circuits.

It is important to note that for $2^{\varepsilon n}$ size and $n$ inputs, Circuit SAT is solvable in polynomial time. Similarly, Circuit SAT is in quasipolynomial time for circuits of size $2^{n^{\varepsilon}}$. Thus Theorems 1.4 and 1.5 are concerned with improving the running time of problems in $\mathrm{P}$ and QP respectively, possibly using nondeterministic algorithms, and the problem GAP $C$ SAT is believed to be in P even for poly $(n)$ circuit sizes.

These new generic algorithm-to-lower-bound connections immediately imply much tighter lower bounds for ACC and ACC $\circ$ THR circuits, namely the separation NQP $\not \subset$ ACC $\circ$ THR:

\footnotetext{
${ }^{3}$ In fact, the GAP $C$ UNSAT algorithm could even use $2^{o(n)}$ bits of advice; see Remark 1.
} 
Theorem 1.6. For every constant $c$, $d$, and $m \geq 2$, there is an $e \geq 1$ and a problem in NTIME $\left[n^{\log ^{e} n}\right]$ which does not have depth$d n^{\log ^{c} n}$-size AC $[m]$ circuits, even with $n^{\log ^{c} n}$ linear threshold gates at the bottom layer.

\subsection{Intuition}

Our proof of the easy witness lemma for NP and NQP has some similarities to the original Impagliazzo-Kabanets-Wigderson argument [12], but makes some important modifications. Let us review their proof. For simplicity, we restrict ourselves here to looking at poly-size circuits, but the full proof is much more general, allowing for quasi-polynomials and even third-exponentials.

IKW's Easy Witness Lemma. At a high level, the proof of Lemma 1.1 works as follows. Assume

(A) NTIME $\left[2^{n}\right]$ has circuits of size $n^{k}$, and

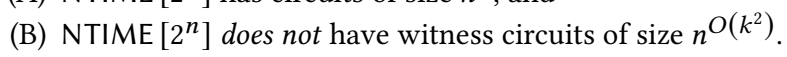

We wish to obtain a contradiction. We show that (A) and (B) contradict the theorem that there is a language $L_{\text {hard }} \in \operatorname{TIME}\left[2^{n^{k+1}}\right]$ such that for all but finitely many $n, L_{\text {hard }}$ does not have $n$-input circuits of size $O\left(n^{k}\right)$ (note this is provable by direct diagonalization; see Theorem 2.6 in the Preliminaries, which shows we can put $L_{h a r d}$ in SPACE $\left[n^{k+1}\right]$ ).

Assumption (A) implies that $L_{\text {hard }} \in \operatorname{TIME}\left[2^{n^{k+1}}\right]$ has $n^{O\left(k^{2}\right)}$. size circuits, by padding/translation. Building on the proof that EXP $\subset \mathrm{P} /$ poly implies EXP $=$ MA ([2]), that in turn implies (as noted by [18]) that $L_{\text {hard }}$ has a Merlin-Arthur protocol $P$ running in $n^{O\left(k^{2}\right)}$ time.

We can use (B) to "derandomize" the Arthur part of the MerlinArthur protocol $P$ for $L_{\text {hard }}$, as follows. Assumption (B) implies that for all $c \geq 1$, there is a "bad" $O\left(2^{n}\right)$-time verifier $V_{c}$ that takes $x$ of length $n$ and $y$ of length $O\left(2^{n}\right)$, such that for infinitely many $n_{i}$, there is a "bad" input $x_{i}$ of length $n_{i}$ such that $V_{c}\left(x_{i}, y_{i}\right)$ accepts for some $y_{i}$, but every $y_{i}$ accepted by $V_{c}\left(x_{i}, \cdot\right)$ has circuit complexity at least $n^{c k^{2}}$. Thus, if we give $x_{i}$ as $n$ bits of advice, nondeterministically guess a string $y_{i}$ of length $O\left(2^{n}\right)$, and verify $V_{c}\left(x_{i}, y_{i}\right)$ accepts, we are guaranteed $y_{i}$ encodes the truth table of a function with circuit complexity at least $n^{c k^{2}}$.

Applying standard hardness-to-randomness connections, the hard function $y_{i}$ can be used to power a pseudorandom generator $G$ with $O\left(k^{2} c \log n\right)$-length seeds that runs in $2^{O(n)}$ time and fools circuits of size $n^{c k^{2} / g}$, for some universal $g \geq 1$. For large enough $c$, we have a generator $G$ that can "fool" the Arthur part of the protocol $P$ for $L_{\text {hard }}$, given an input and Merlin's message. So by guessing $y_{i}$ (of $O\left(2^{n}\right)$ length), guessing Merlin's message, and running $G$ on all seeds, the Merlin-Arthur protocol $P$ can be faithfully simulated on infinitely many input lengths $n_{i}$ by a nondeterministic algorithm $N$, running in $2^{O(n)}$ time, with $n$ bits of advice. Finally, applying (A) once more to $N$, we conclude that $L_{\text {hard }}$ has circuits of size $O\left(n^{k}\right)$ on infinitely many input lengths, contradicting the definition of $L_{\text {hard }}$.

How to Improve IKW? A Minor Modification. Suppose we replace NTIME $\left[2^{n}\right]$ with NP or NQP in assumptions (A) and (B) in the above. What goes wrong? For starters, we no longer have $\mathrm{EXP}=\mathrm{MA}$ or PSPACE $=\mathrm{MA}$ as a consequence of our assumptions, so we can no longer easily infer from our assumptions that a hard language $L_{\text {hard }}$ has efficient Merlin-Arthur protocols! Thus it is not at all clear what lower bound we may hope to contradict. All is not lost though. Since [12] appeared, other circuit lower bounds have been proved, such as the celebrated result of Santhanam [20] that for all $k, \mathrm{MA} / 1 \not \subset \mathrm{SIZE}\left[n^{k}\right]$. In particular, Santhanam ([20], Theorem 4.3) gives an explicit language $L_{k}$ computable by a Merlin-Arthur protocol $P_{k}$ in $n^{O\left(k^{2}\right)}$ time (with 1 bit of advice) which does not have $n^{k}$-size circuits. Letting $d \gg k^{2}$, and assuming NTIME $\left[n^{d}\right]$ does not have witness circuits of $n^{O\left(k^{2}\right)}$ size, we can derandomize $P_{k}$ just as in the IKW argument, by guessing-and-verifying a witness of length $O\left(n^{d}\right)$ with circuit complexity at least $n^{\Omega\left(k^{2}\right)}$. We conclude that $L_{k}$ is solvable in nondeterministic $n^{O(d)}$ time, with $n+1$ bits of advice, for infinitely many input lengths. If we further assume that NTIME $\left[n^{O(d)}\right]$ has $n^{k}$-size circuits, we could then infer that $L_{k}$ has $O(n)^{k}$-size circuits for infinitely many input lengths. Unfortunately, Santhanam's MA/1 lower bound for $L_{k}$ is not known to hold in the infinitely-often setting; his proof only rules out a $n^{k}$-size circuit family computing $L_{k}$ on all but finitely many input lengths. However, this new argument does show that, if NP has $n^{k}$-size circuits, then NP also has $n^{O\left(k^{2}\right)}$-size witness circuits for infinitely many input lengths $n$ (because if NP did not have such witness circuits almost everywhere, then we could simulate $P_{k}$ nondeterministically almost everywhere with small advice, and get the desired contradiction). Call this the "infinitely-often witness" argument.

As far as we can tell, having witness circuits "infinitely often" is not enough for the lower bound applications. (For one, we would need an "almost-everywhere" nondeterministic time hierarchy theorem to get the final contradiction, but it is open whether NEXP is contained in io-NP!) We need another approach to getting a contradiction; perhaps another type of circuit lower bound for MA would work?

Our Approach: "Almost" an Almost-Everywhere Circuit Lower Bound for MA. Again, here we stick to polynomial-size circuits, to keep the exposition simple. By allowing a little more advice to a Merlin-Arthur protocol, we still do not get a lower bound for all but finitely many input lengths, but we can guarantee that MA/O $(\log n)$ has $n^{k}$-size lower bounds on input lengths that are only polynomially far apart, for all but finitely many $n$. In particular, we prove:

Theorem 1.7 (INFormal). For all $k$, there is an explicit language $L_{k}^{\prime}$ computable by a Merlin-Arthur protocol $P_{k}$ in $n^{O\left(k^{2}\right)}$ time (with $O(\log n)$ bits of advice) such that, for all but finitely many input lengths $n$, either

- $L_{k}^{\prime}$ does not have an $n^{k}$-size circuit on inputs of length $n$, or

- $L_{k}^{\prime}$ does not have an $n^{O\left(k^{2}\right)}$ size circuit on inputs of length $n^{O(k)}$.

The full formal statement can be found in Theorem 3.1. Our $L_{k}^{\prime}$ is related to Santhanam's aforementioned MA/1 language, as 
they both attempt to compute a paddable, self-correctable, and downward self-reducible PSPACE-complete language LPSPACE on padded inputs (due to Trevisan and Vadhan [23], with modifications by Santhanam [20]). Santhanam breaks his analysis into two cases, whether or not PSPACE has polynomial-size circuits, and shows his language does not have small circuits in either case.

Our advice and analysis are very different. At a high level, our Merlin-Arthur protocol $P_{k}$ has two parts. Its advice on input length $n$ encodes the largest $\ell_{n}$ such that $L_{\text {PSPACE }}$ has circuits of size $n^{a k^{2}}$ on length- $\ell_{n}$ inputs for a sufficiently large $a \geq 1$, which will indicate which part to run on an input $y$ of length $n$ :

Part 1: If $\ell_{n} \geq n^{c k}$, then $P_{k}$ guesses-and-randomly-checks a circuit of $n^{a k^{2}}$ size with $n^{c k}$ inputs for LPSPACE (via Theorem 2.5), and uses this circuit to decide if $y$ is in a language $L_{\text {diag }} \in$ SPACE $\left[n^{O(k)}\right]$ (Theorem 2.6) which provably does not have $n^{k}$-size circuits for any infinite set of input lengths. (Here, we use the fact that $L_{\text {PSPACE }}$ is PSPACE-complete.)

Part 2: If $\ell_{n}<n^{c k}$, then $P_{k}$ parses $y$ as a padded string $1^{q_{0 x}}$. If $|x|+1>\ell_{n}$ then $P_{k}$ rejects, else $P_{k}$ tries to compute $L_{\text {PSPACE }}$ on $1^{\ell_{n}-|x|-1} 0 x$, by guessing-and-checking a circuit with $\ell_{n}$ inputs and $n^{a k^{2}}$ size.

(Here, $c \leq a$ is a large enough constant.) Let $L_{k}^{\prime}$ be the language accepted by $P_{k}$. By our choice of $\ell_{n}, P_{k}^{\prime}$ only guesses circuits for LPSPACE when valid ones exist, and it uses the circuit as an oracle in Santhanam's checker for LPSPACE. Together, these ensure that $P_{k}^{\prime}$ satisfies the MA promise condition on all inputs.

To prove the lower bound for $L_{k}^{\prime}$, suppose there is an infinite set $S \subseteq \mathbb{N}$ such that for all $n \in S$,

(A) $L_{k}^{\prime}$ has $n^{k}$-size circuits for length- $n$ inputs, and

(B) $L_{k}^{\prime}$ has $n^{c k^{2}}$-size circuits for length- $n^{c k}$ inputs.

We wish to derive a contradiction. We consider two cases:

Case 1. Suppose there is an infinite subset $S^{\prime} \subseteq S$ such that for all $n \in S^{\prime}, \ell_{n^{c k}} \geq n^{c^{2} k^{2}}$. Then for all $n \in S^{\prime}, P_{k}$ runs the first part of its protocol on $n^{c k}$-length inputs. In particular, for all $n \in S^{\prime}$, $P_{k}$ on $y$ of length $n^{c k}$ guesses a circuit of $n^{O\left(k^{3}\right)}$ size with $n^{O\left(k^{2}\right)}$ inputs for $L_{\text {PSPACE}}$, verifies the circuit using randomness, then uses the circuit to simulate $L_{\text {diag }}$. By definition of $L_{\text {diag }}$, for almost all $n \in S^{\prime}, L_{k}^{\prime}$ does not have $n^{c k^{2}}$-size circuits for $n^{c k}$-length inputs. However, note this is in direct contradiction with (B).

Case 2. Suppose for all but finitely many $n \in S, \ell_{n^{c k}}<n^{c^{2} k^{2}}$. Then for almost all $n \in S$, the protocol $P_{k}$ runs the second part of its protocol on $n^{c k}$-length inputs: $P_{k}$ simulates $L_{\text {PSPACE }}$ on $1^{\ell_{n}-|x|-1} 0 x$, provided $|x|+1 \leq \ell_{n}$. We show how the second part of $P_{k}$, together with assumptions (A) and (B), can be used to infer that LPSPACE itself has an $n^{c k^{2}}$-size circuit on inputs of length $n^{c k}$, using a "bootstrapping" kind of argument.

In more detail, assume for some $m$ that $L_{k}^{\prime}$ has an $m^{k}$-size circuit on length- $m$ input, and that $\ell_{m}<m^{c k}$, so that the second part of $P_{k}$ is run on length- $m$ inputs. We start by observing $\ell_{m} \geq 1$ : $P_{k}$ can (unconditionally) guess-and-check $O(1)$-size circuits for $L_{\text {PSPACE }}$ on inputs of length 1 . If $P_{k}$ on $m$-bit inputs can be used to compute $L_{\text {PSPACE }}$ on $\ell$-bit inputs (i.e., $m^{c k}>\ell_{m} \geq \ell$ ), then (using the assumed $L_{k}^{\prime}$ circuit) there is an $m^{k}$-size circuit for $L_{\text {PSPACE }}$ on $\ell$-bit inputs. By the downward self-reducibility of $L_{\text {PSPACE}}$, there is also an $m^{d k}$-size circuit $C$ computing LPSPACE on $(\ell+1)$-bit inputs, for some (fixed) $d$. For $d \leq a k$, this circuit $C$ implies that $\ell_{m} \geq \ell+1$. Thus the protocol $P_{k}$ has enough time to guess and verify this $C$, and the second part of $P_{k}$ on $m$-bit inputs can also compute $L_{\text {PSPACE }}$ on $(\ell+1)$-bit inputs. Since $L_{k}^{\prime}$ has a $m^{k}$-size circuit on length- $m$ inputs, the $m^{d k}$-size circuit for LPSPACE on $(\ell+1)$-bit inputs can then be replaced by an $m^{k}$-size circuit(!). We can repeat this argument for lengths $\ell=1, \ldots, m-1$, implying that $L_{\text {PSPACE }}$ has $m^{k}$-size circuits for input length $m$.

Therefore, in Case 2, for almost all $n \in S, n^{c k^{2}}$-size circuits for $L_{k}^{\prime}$ on length- $n^{c k}$ inputs (assumption (B)) implies that $L_{\text {PSPACE }}$ has an $n^{c k^{2}}$-size circuit for length $n^{c k}$. Since $c \leq a$, by definition of $\ell_{n}$ it follows that for almost all $n \in S$ we have $\ell_{n} \geq n^{c k}$. Thus the first part of $P_{k}$ is run on these $n \in S$, so $P_{k}$ is simulating $L_{\text {diag }}$ on almost all inputs of length $n \in S$. By definition of $L_{\text {diag }}$, for almost all $n \in S, L_{k}^{\prime}$ does not have $n^{k}$-size circuits for length- $n$ inputs. But this is a direct contradiction with (A). This completes the intuition for Theorem 1.7.

From an MA Circuit Lower Bound to Easy Witnesses. Let us briefly discuss how this Merlin-Arthur lower bound can be used for our new Easy Witness Lemma. We have to show the "almost" almosteverywhere lower bound is enough for obtaining a contradiction with the aforementioned "infinitely-often witness" argument. Assume NP has $n^{k}$-size circuits, and assume there is an NP-verifier $V$ that does not have $n^{c k^{3}}$-size witness circuits (rather than $n^{k^{2}}$, as in IKW) for large $c$. Then there are infinitely many "bad" inputs $x_{i}$ such that $V_{c}\left(x_{i}, y_{i}\right)$ accepts for some $y_{i}$, but every $y_{i}$ accepted by $V_{c}\left(x_{i}, \cdot\right)$ has circuit complexity at least $n^{c k^{3}}$. Then, just as in the IKW argument and infinitely-often witness argument, we can use $y_{i}$ in a pseudorandom generator that fools circuits of size $n^{c k^{3} / g}$. Take the "hard" Merlin-Arthur protocol $P_{k}$. On infinitely many pairs of input lengths $\left(n, n^{\Theta(k)}\right)$ corresponding to the bad inputs $x_{i}$, we want to derive an $n^{k}$-size circuit for length- $n$ inputs and an $n^{\Theta\left(k^{2}\right)}$-size circuit on length- $n^{\Theta(k)}$ inputs, to contradict the "almost" almost-everywhere lower bound. Because the circuit constructed from the Merlin-Arthur protocol has size $n^{O\left(k^{3}\right)}$ even on inputs of length $n^{\Theta(k)}$, this pseudorandom generator works not only for input length $n$ but also for length $n^{k}$. Thus, if witness circuits of size $n^{c k^{3}}$ did not exist, we would be able to simulate $P_{k}$ on infinitely many pairs of input lengths $\left(n, n^{\Theta(k)}\right)$ in NP, using $n+O(\log n)$ bits of advice. Assuming NP has $n^{k}$-size circuits, this contradicts the earlier lower bound proved for $P_{k}$.

Outline of the Rest. The rest of the paper is structured as follows. In Section 2 we give definitions and review related material from the literature. In Section 3 we prove the "almost" almost-everywhere lower bound for MA. In Section 4 we prove the new Easy Witness Lemma in its full form. In Section 5 we improve connections between circuit-analysis and circuit lower bounds using the Lemma. In Section 6 we conclude with some open questions. 


\section{PRELIMINARIES}

Basics. All languages/functions are over $\{0,1\}$, and all logarithms are base-2 with ceilings as appropriate. We assume knowledge of basic complexity theory [1] such as circuit families computing a language, and complexity classes such as EXP, NEXP, ACC, etc. We use SIZE $[s(n)]$ to denote the class of problems computed by a (non-uniform) $s(n)$-size circuit family. When we refer to a "typical" circuit class ( $\mathrm{AC}^{0}, \mathrm{ACC}, \mathrm{TC}^{0}, \mathrm{NC}^{1}, \mathrm{NC}$, or P/poly), we assume the underlying circuit families are non-uniform and of polynomial-size, unless otherwise specified.

We will often take as given that any $O(t(n))$-time algorithm can be converted (in $O\left(t(n)^{2}\right)$ time) into an equivalent circuit family of size at most $t(n)^{2}$, for all but finitely many $n$. (Different circuit conversions would only affect the constant factors in our arguments.)

We use advice classes: for a deterministic or nondeterministic class $C$ and a function $a(n), C / a(n)$ is the class of languages $L$ such that there is an $L^{\prime} \in C$ and an arbitrary function $f: \mathbb{N} \rightarrow\{0,1\}^{\star}$ with $|f(n)| \leq a(n)$ for all $x$, such that $L=\left\{x \mid(x, f(|x|)) \in L^{\prime}\right\}$. That is, the arbitrary advice string $f(n)$ can be used to solve all $n$-bit instances within class $C$.

In our MA lower bound, we need to show that some protocol "satisfies the MA promise" on all inputs:

Definition 2.1. We say that a Merlin-Arthur protocol $P$ satisfies the MA promise on length $\ell$ if for all $x \in\{0,1\}^{\ell}$, either there is a Merlin message such that Arthur accepts $x$ with probability at least $2 / 3$, or for all Merlin messages, Arthur rejects $x$ with probability at least $2 / 3$. In other words, $P$ satisfies the MA promise on length $\ell$ if it obeys the usual semantics of an MA language on all inputs of that length.

Typical Circuit Classes. In general, a Boolean circuit class $C$ is a collection of descriptions of Boolean functions. $C$ is evaluatable if given a description of a $C$ from $C$ and given an $0 / 1$ assignment $a$ to some (but not necessarily all) of the inputs, the sub-circuit $C(a)$ can be determined in polynomial time. Say that a class of Boolean circuits $C$ is closed under negation if there is a poly $(n)$-time algorithm $A$ such that, given any $n$-bit description of a circuit $C$ from $C, A$ outputs a description of $\neg C$. $C$ is closed under conjunctions if there is a poly $(n)$-time algorithm $A$ such that, given the descriptions of $n$ circuits $C_{1}, C_{2}, \ldots, C_{n}$, each on $n$ inputs from $C$, algorithm $A$ outputs a circuit $C^{\prime}\left(x_{1}, \ldots, x_{n}\right) \equiv C_{1}\left(x_{1}, \ldots, x_{n}\right) \wedge \cdots \wedge C_{n}\left(x_{1}, \ldots, x_{n}\right)$. Any $C$ satisfying all three properties is called typical.

Circuit Complexity of Strings and Pseudorandom Generators. Let $x_{1}, \ldots, x_{2} \ell$ be the $\ell$-bit strings in lexicographical order. For a circuit $C$ on $\ell$ inputs, define $t t(C):=C\left(x_{1}\right) \cdots C\left(x_{2} \ell\right)$, i.e., $t t(C) \in$ $\{0,1\}^{2^{\ell}}$ is the truth table of $C$. For every string $y$, let $2^{\ell}$ be the smallest power of 2 such that $2^{\ell} \geq|y|+1$. We define the circuit complexity of $y$, or $C C(y)$, to be the circuit complexity of the $\ell$-input function defined by the truth table $y 10^{2^{\ell}-|y|-1}$. We will use the following strong construction of pseudorandom generators from hard functions:
THEOREM 2.2 (UMANS [24]). There is a universal constant $g$ and $a$ function $G:\{0,1\}^{\star} \times\{0,1\}^{\star} \rightarrow\{0,1\}^{\star}$ such that, for all $s$ and $Y$ satisfying $C C(Y) \geq s^{g}$, and for all circuits $C$ of size s,

$$
\left|\operatorname{Pr}_{x \in\{0,1\}^{g \log |Y|}}[C(G(Y, x))=1]-\operatorname{Pr}_{x \in\{0,1\}^{s}}[C(x)=1]\right|<1 / s .
$$

Furthermore, $G$ is computable in poly $(|Y|)$ time.

Witness Circuits. We formally define the terminology behind "witness circuits" (also found in [25, 27, 28]).

Definition 2.3. Let $L \in \mathrm{NTIME}[t(n)]$ where $t(n) \geq n$ is constructible. An algorithm $V(x, y)$ is a verifier for $L$ if $V$ runs in time $O(|y|+t(|x|))$ and for all strings $x$,

$$
\begin{aligned}
x \in L \Longleftrightarrow & \text { there is a } y \text { of length } O(t(n)) \text { (a witness } \\
& \text { for } x \text { ) such that } V(x, y) \text { accepts. }
\end{aligned}
$$

We denote $L(V)$ to be the NTIME $[t(n)]$ language verified by $V$. We say $V$ has witness circuits of size $w(n)$ if for all strings $x$, if $x \in L(V)$ then there is a $y_{x}$ of length $O(t(n))$ such that $V\left(x, y_{x}\right)$ accepts and $y_{x}$ has circuit complexity at most $w(n)$.

We say $L$ has witness circuits of size $w(n)$ if every verifier for $L$ has witnesses of size $w(n)$.

NTIME $[t(n)]$ has witness circuits of size $w(n)$ if every language $L \in \operatorname{NTIME}[t(n)]$ has witness circuits of size $w(n)$.

Thinking of $w(n) \ll t(n)$, if a nondeterministic time- $t(n)$ language $L$ has size- $w(n)$ witness circuits, then for every

$O(t(n))$-time verifier $V$ for $L$, all $x \in L$ have a highly compressible witness $y_{x}$ with respect to $V: y_{x}$ is a string of length $t(n)$, but as a truth table, it has a circuit of size at most $w(n)$.

A Structured PSPACE-Complete Problem. A fundamental result used often in the theory of pseudorandomness (modifying a construction of Trevisan and Vadhan [20,23]) is that there is a PSPACEcomplete language with strong reducibility properties. First, we define the properties.

Definition 2.4. $L \subseteq\{0,1\}^{\star}$ is downward self-reducible if there is a (deterministic) polynomial-time oracle algorithm $A^{\text {? }}$ such that for all $n$ and all $x \in\{0,1\}^{n}, A^{L^{n-1}}(x)=L(x)$.

THEOREM 2.5 ([20]). There is a PSPACE-complete language $L_{\mathrm{PSPACE}}$ which is paddable, downward self-reducible, and furthermore is "checkable" in that there is a probabilistic polynomial-time oracle Turing machine $M$ so that, for any input $x$,

- $M$ asks its oracle queries only of length $|x|$.

- if $M$ is given $L_{\mathrm{PSPACE}}$ as oracle and $x \in L$, then $M$ accepts with probability 1.

- if $x \notin L_{\mathrm{PSPACE}}$, then irrespective of the oracle given to $M, M$ rejects with probability at least $2 / 3$.

Santhanam proves the existence of the probabilistic poly-time "checker" $M$, and that LPSPACE is PSPACE-complete; we also note in the below proof that his language is paddable and downward self-reducible. All four of these properties of LPSPACE will be used in our argument.

Proof. We simply verify that the language given by Santhanam [20] has the additional desired properties. Let

$L_{\text {PSPACE }}=\left\{1^{i} \mid i \geq 0\right\} \cup\left\{1^{i} 0 x \mid x \in S\right.$ and $\left.i \geq 0\right\}$, where $S$ is the 
PSPACE-complete language of Trevisan and Vadhan [23] which is downward self-reducible and "self-correctable" (which we do not define here). By definition, it is clear that for any amount of padding $m, 1^{m} x \in L_{\text {PSPACE }} \Longleftrightarrow x \in L_{\text {PSPACE}}$. To construct a downward self-reduction $A$ for $L_{P S P A C E}$, we can make use of the downward self-reduction $A_{0}$ for the language $S$ :

$A$ : On input $y$, parse $y=1^{m} 0 x$.

If $m>0$, accept iff $1^{m-1} 0 x \in L_{\mathrm{PSPACE}}$.

If $m=0$, run the downward self-reduction $A_{0}$ on $x$.

For every query $x^{\prime}$ for $S$ made by $A_{0}$, query $0 x^{\prime} \in L_{\text {PSPACE }}$ instead.

Accept if and only if $A_{0}$ accepts on $x$.

Observe $A$ is a downward self-reduction for LPSPACE: Either $y$ has some amount of 1-padding at the beginning, or it has no 1padding. In the first case, $L_{\text {PSPACE }}$ can be quickly solved with one oracle query (remove a 1). In the second case, the downward selfreduction for $S$ can be used. Since $x$ has length $|y|-1$, all of the queries $x^{\prime}$ made by $A_{0}$ are of length $|y|-2$, so $0 x^{\prime}$ is a query to $L_{\text {PSPACE }}$ of length $|y|-1$.

We also need a standard almost-everywhere circuit lower bound, provable by direct diagonalization:

Theorem 2.6. Let $s(n)<2^{n} /(2 n)$ be space constructible. Then there is a language $L_{\text {diag }} \in \operatorname{SPACE}\left[s(n)^{2}\right]$ that does not have $s(n)$ size circuits for all but finitely many input lengths $n$.

Proof. Folklore. On an input of length $n$, using $O\left(s(n)^{2}\right)$ space one can exhaustively try all Boolean functions

$f:\{0,1\}^{2 \log (s(n))} \rightarrow\{0,1\}$ and circuits of size $s(n)$, until one finds the lexicographically first function $f_{n}$, represented as a bit string of length $2^{2 \log (s(n))} \leq O\left(s(n)^{2}\right)$, that is not computable by any circuit of size $s(n)$. Then the padded language

$$
L_{\text {diag }}=\bigcup_{n \in \mathbb{N}}\left\{1^{n-1-2 \log (s(n))} 0 x|| x \mid=2 \log (s(n)) \wedge f_{n}(x)=1\right\}
$$

does not have $s(n)$-size circuits for all but finitely many $n$, but is computable in space $O\left(s(n)^{2}\right)$.

Significant Separations. For the case of poly-size circuits, the circuit lower bound of Theorem 3.1 implies a "significant separation", as defined by Fortnow and Santhanam [10]. They say a class $C$ has a significant separation from $\mathcal{D}$, if there is an $f \in C$ such that for all $g \in \mathcal{D}$ there is a $k$ so that for every $m$, the function $f_{n}$ (restricted to $n$-bit inputs) differs from $g_{n}$ on some input length $n$ in $\left[m, m^{k}\right]$. The lower bound of Theorem 3.1 implies a significant separation of MA (with advice) from small circuits. However, our lower bound seems a bit stronger in that, rather than proving an lower bound for some input length in all polynomially-long intervals, we show an lower bound for every pair of input lengths which are polynomially far apart.

\section{3 “ALMOST” AN ALMOST-EVERYWHERE CIRCUIT LOWER BOUND FOR MA}

In this section, we prove the main lower bound needed for our new Easy Witness Lemmas. For a language $L \subseteq\{0,1\}^{\star}$ and $n \in \mathbb{N}$, let $L^{n}$ be the language $L$ restricted to $n$-bit inputs. Say that $s: \mathbb{N} \rightarrow \mathbb{N}$ is a circuit-size function if it is increasing, time constructible, and $s(n)<2^{n} /(2 n)$ holds for all sufficiently large $n$.

Theorem 3.1 ("Almost” A.E. Circuit Lower Bound For MA WITH ADVICE). There are $d_{1}, d_{2}, d_{3} \geq 1$ such that for all circuit-size functions $s$ and time-constructible $s_{1}, s_{2}: \mathbb{N} \rightarrow \mathbb{N}$ satisfying

(i) $s_{2}(n) \geq s(n)^{2 d_{2}}$,

(ii) $s_{1}(n) \geq s\left(s_{2}(n)\right)$, and

(iii) $s_{1}(n) \geq s(n)^{2 d_{1}+1}$,

there is a language $L_{1}$ computable by a Merlin-Arthur protocol in $O\left(s_{1}(n)^{2} \cdot s_{2}(n)^{d_{3}}\right)$ time with $2 \log s_{2}(n)$ advice, such that for all sufficiently large $n \in \mathbb{N}$, either

- the circuit complexity of $L_{1}^{n}$ is greater than $s(n)$, or

- the circuit complexity of $L_{1}^{s_{2}(n)}$ is greater than $s\left(s_{2}(n)\right)$.

That is, we "almost" prove an almost-everywhere size-s $(n)$ circuit lower bound for MA with $2 \log s(n)$ advice: for almost all $n$, the MA language $L_{1}$ either has high complexity on length $n$, or it has high complexity on length $s_{2}(n)$. Later, we will use this "almost" almost-everywhere lower bound to obtain a stronger easy witness lemma.

The rest of this section is devoted to proving Theorem 3.1. The language $L_{1}$ is formed from two different Merlin-Arthur protocols:

(1) One protocol attempts to solve the problem $L_{\text {diag }}$ that does not have size-s $(n)$ circuits almost everywhere (from Theorem 2.6), by guessing and checking circuits for the complete language $L_{P S P A C E}$, and applying a reduction from $L_{\text {diag }}$ to LPSPACE.

(2) The other protocol solves LPSPACE (from Theorem 2.5) on inputs with sufficiently long padding.

The $2 \log s_{2}(n)$ bits of advice are used to determine which of these two languages can be successfully computed by our MA protocol, on a given input length.

Set $d_{1}, d_{2}, d_{3} \geq 1$ such that:

- The downward self-reduction $A$ for $L_{\text {PSPACE }}$ (as seen in Theorem 2.5) runs in time $O\left(n^{d_{1}}\right)$.

- For every language $L \in \operatorname{SPACE}[s(n)]$, there is a reduction from $L$ to $L_{\text {PSPACE }}$ which runs in $O\left(s(n)^{d_{2}}\right)$ time and produces an $L_{\text {PSPACE }}$ instance of length $s(n)^{d_{2}}$. To ensure this condition, set $d_{2}$ such that the language

$L^{\prime}=\left\{\left(M, x, 1^{t}\right) \mid M\right.$ accepts $x$ using space at most $\left.t\right\}$

can be reduced to $L_{\text {PSPACE }}$ in $O\left(n^{d_{2}-1}\right)$ time. Then, every SPACE $[s(n)]$ language $L$ has an $O(s(n))$-time reduction to $L^{\prime}$, and therefore $L$ has an $O\left(s(n)^{d_{2}}\right)$-time reduction to $L_{\text {PSPACE}}$.

- The probabilistic polytime oracle machine $M$ for $L_{\text {PSPACE }}$ (as seen in Theorem 2.5) runs in time $O\left(n^{d_{3}}\right)$.

Since $s(n)<2^{n} /(2 n)$, there is a language $L_{\text {diag }}$ without $s(n)$ size circuits almost everywhere (Theorem 2.6). Using $L_{\text {diag }}$ (which 
is in SPACE $\left.\left[s(n)^{2}\right]\right)$ and the reduction from $L_{\text {diag }}$ to $L_{\text {PSPACE, we }}$ have:

CLAIM 1. There is an $O\left(s(n)^{2 d_{2}}\right)$-time reduction $R$ such that for all strings $y,|R(y)|=s(|y|)^{2 d_{2}}$ and $y \in L_{\text {diag }} \Longleftrightarrow R(y) \in L_{\text {PSPACE. }}$

We are now in position to define a Merlin-Arthur protocol $M_{1}$ recognizing a hard language $L_{1}$. For input length $n$, define

$$
\begin{array}{r}
\ell(n):=\text { the largest integer } \ell \text { such that } L_{\mathrm{PSPACE}}^{\ell} \\
\text { has a circuit of size at most } s_{1}(n) .
\end{array}
$$

Note that a circuit always exists when $s_{1}(n) \geq 2^{\ell}$, so we have $\ell(n) \geq \log \left(s_{1}(n)\right)$. Also note that since $L_{\text {PSPACE }}$ is paddable, a circuit for $L_{\text {PSPACE }}^{m}$ can solve $L_{\text {PSPACE }}^{\ell}$ for any $\ell<m$ by setting the first $m-\ell$ bits to 1 . Our advice $\alpha_{n}$ for inputs of length $n$ will be the integer

$$
\alpha_{n}:=\min \left\{s_{2}(n), \ell(n)\right\} .
$$

Note that $\alpha_{n}$ can be encoded in at most $2 \log s_{2}(n)$ bits. (For example, the first bit of the encoding could denote which of $s_{2}(n), \ell(n)$ is the minimum; if it is $s_{2}(n)$, then no further information is required, as that can be computed by the machine. If the minimum is $\ell(n)$, then we can encode $\ell(n)$ as an integer in $\left\{0,1, \ldots, s_{2}(n)-1\right\}$ using less than $2 \log s_{2}(n)$ bits.)

Let $R$ be the reduction from $L_{\text {diag }}$ to $L_{\text {PSPACE }}$ of Claim 1, with running time $O\left(s(n)^{2 d_{2}}\right)$. The Merlin-Arthur protocol $M_{1}$ defining a language $L_{1}$ is described in pseudocode as follows:

$$
\begin{aligned}
& \text { Advice } \alpha_{n}:=\min \left\{s_{2}(n) \text {, largest } \ell \in \mathbb{N} \text { s.t. } L_{\text {PSPACE }}^{\ell}\right. \text { has } \\
& \text { an } \left.s_{1}(n) \text {-size circuit }\right\} \\
& M_{1}(y)_{/ \alpha_{n}}:=\text { Let } n=|y| \text {. } \\
& \text { If } \alpha_{n}=s_{2}(n) \text { : } \\
& \text { // simulate } L_{\text {diag }} \text { on } y \\
& \text { Let } z=R(y) \text {; note }|z| \leq s_{2} \text { (n) (by constraint (i)). } \\
& \text { Guess an } s_{2}(n) \text {-input circuit } C \text { of size } s_{1}(n) \text {. } \\
& \text { Output } M^{C}\left(1^{s_{2}(n)-|z|} z\right) \text { (Theorem 2.5). } \\
& \text { Otherwise }\left(\alpha_{n}<s_{2}(n)\right) \text { : } \\
& \text { // simulate } L_{P S P A C E} \text { on a padded input } \\
& \text { Parse } y=1^{a} 0 x \text { for some } a \geq 0 \text {. } \\
& \text { If }|x|+1>\alpha_{n} \text { then reject. } \\
& \text { Guess an } \alpha_{n} \text {-input circuit } C \text { of size } s_{1}(n) \text {. } \\
& \text { Output } M^{C}\left(1^{\alpha_{n}-|x|-1} 0 x\right) \text { (Theorem 2.5). }
\end{aligned}
$$

MA Promise and Running Time Analysis. First, we verify that the advice $\alpha_{n}$ ensures that $M_{1}$ satisfies the MA promise on all input lengths - in particular, $\alpha_{n}$ ensures that the desired circuits $C$ being guessed always exist in both branches of $M_{1}$ - and that $M_{1}$ runs in $O\left(s_{1}(n)^{2} \cdot s_{2}(n)^{d_{3}}\right)$ time. Intuitively, Merlin provides a circuit $C$ and Arthur runs $M^{C}$.
- If $\alpha_{n}=s_{2}(n)$, then by definition (2) (and padding) $L_{\text {PSPACE }}^{s_{2}(n)}$ has a $s_{1}(n)$-size circuit.

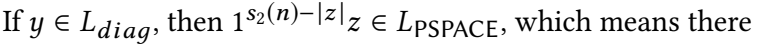
is a (Merlin) circuit $C$ of size $s_{1}(n)$ such that $M^{C}(z)$ (Arthur) accepts with probability 1 (following Theorem 2.5). In particular, the reduction from $L_{\text {diag }}$ to $L_{\text {PSPACE }}$ runs in time $O\left(s(n)^{2 d_{2}}\right)$ and produces an instance $z$ of length $s(n)^{2 d_{2}}$. By constraint (i) $\left(s_{2}(n) \geq s(n)^{2 d_{2}}\right)$, we can pad $z$ and $M^{C}$ will accept it with probability 1 .

If $y \notin L_{\text {diag }}$, then for every possible (Merlin) circuit $C$, the probabilistic polynomial time $M^{C}$ (Arthur) will reject with probability at least $2 / 3$ (again following Theorem 2.5). So $M_{1}$ satisfies the MA promise in this case. Simulating $M^{C}$ can be done in time $O\left(s_{1}(n)^{2} \cdot s_{2}(n)^{d_{3}}\right)$, where the extra $s_{1}(n)^{2}$ factor comes from evaluating the circuit $C$ on each oracle query.

- If $\alpha_{n}<s_{2}(n)$, then $M_{1}$ guesses a $s_{1}(n)$-size circuit $C$ for $L_{\mathrm{PSPACE}}^{\alpha_{n}}$, then runs $M^{C}\left(1^{\alpha_{n}-|x|-1} 1 x\right)$.

Suppose $y=1^{a} 0 x \in L_{\text {PSPACE }}$. By the paddability of $L_{\text {PSPACE}}$, it follows that $1^{\alpha_{n}-|x|-1} 0 x \in L_{\text {PSPACE }}$ as well. Moreover by definition (2), $L_{\text {PSPACE }}^{\alpha_{n}}$ has a circuit of size $s_{1}(n)$ when $\alpha_{n}<s(n)$. Therefore there is a circuit $C$ of size$s_{1}(n)$ and $\alpha_{n}$ inputs such that $M^{C}\left(1^{\alpha_{n}-|x|-1} 0 x\right)$ accepts with probability 1 (following Theorem 2.5).

On the other hand, if $y=1^{a} 0 x \notin L_{\mathrm{PSPACE}}$, then we also have $1^{\alpha_{n}-|x|-1} 0 x \notin L_{\text {PSPACE}}$. By the properties of $M$ from Theorem 2.5, it follows that for every possible circuit $C$, $M^{C}\left(1^{\alpha_{n}-|x|-1} 0 x\right)$ rejects with probability at least $2 / 3$, so $M_{1}$ satisfies the MA promise in this case as well.

Here, the running time of $M_{1}$ is the time needed to run $M^{C}\left(1^{\alpha_{n}-|x|-1} 0 x\right)$ by directly simulating $C$, which is $O\left(s_{1}(n)^{2} \cdot \alpha_{n}^{d_{3}}\right) \leq O\left(s_{1}(n)^{2} \cdot s_{2}(n)^{d_{3}}\right)$.

Therefore $M_{1}$ with advice $\left\{\alpha_{n}\right\}$ satisfies the MA promise on all inputs and runs in $O\left(s_{1}(n)^{2} \cdot s_{2}(n)^{d_{3}}\right)$ time.

"Almost" Almost-Everywhere Hardness. Let $L_{1}$ be the language recognized by $M_{1}$. Next we show for all sufficiently large $n \in \mathbb{N}$, either the circuit complexity of $L_{1}^{n}$ is greater than $s(n)$, or the circuit complexity of $L_{1}^{s_{2}(n)}$ is greater than $s\left(s_{2}(n)\right)$. We do this by showing that if the circuit complexity was low in both cases, even infinitely often, then the language $L_{\text {diag }}$ would also have size-s $(n)$ circuits infinitely often, contradicting Theorem 2.6.

For the sake of contradiction, assume there is an infinite sequence $\left\{n_{i}\right\}$ such that

(A) $L_{1}^{n_{i}}$ has a circuit of size $s\left(n_{i}\right)$ and

(B) $L_{1}^{s_{2}\left(n_{i}\right)}$ has a circuit of size $s\left(s_{2}\left(n_{i}\right)\right)$.

Let $I=\left\{n_{i} \mid i \in \mathbb{N}\right\} \cup\left\{s_{2}\left(n_{i}\right) \mid i \in \mathbb{N}\right\}$. We begin by showing that for all but finitely many $m \in I$, if $L_{1}$ has $s(m)$-size circuits on length $m$ inputs, then $L_{1}$ cannot be simulating $L_{\text {diag }}$ on length $m$ (therefore it must be simulating the padded version of $L_{\text {PSPACE}}$, instead). We use the abbreviation "a.e." to mean "almost every", a.k.a. "all but finitely many" in the following. 
Proposition 3.2. For a.e. $m \in I$, if $L_{1}^{m}$ has a $s(m)$-size circuit, then $\ell(m)<s_{2}(m)$.

Proof. Suppose there were infinitely many $m \in I$ such that $L_{1}^{m}$ has a circuit of size $s(m)$ and $\ell(m) \geq s_{2}(m)$. Then $\alpha_{m}=s_{2}(n)$ for those $m$, and by the definition of $L_{1}, L_{1}^{m}=L_{d i a g}^{m}$ for those $m$. Thus $L_{\text {diag }}$ would have circuits of size $s(m)$ for infinitely many $m$, which is a contradiction to Theorem 2.6.

Applying assumption (A) to Proposition 3.2, we find that for all sufficiently large $m \in I$,

$$
\alpha_{m}<s_{2}(m) \text {. }
$$

Thus for all sufficiently large $m \in I$, a circuit for $L_{1}^{m}$ computes a padded version of $L$ PSPACE, under our assumptions. Our next step is to show that sufficiently small circuits for $L_{1}$ for a given input length $m$ actually implies analogously small circuits for $L_{P S P A C E}$ on input length $m$ :

Lemma 3.3. For a.e. $m \in I$, if $L_{1}^{m}$ has a size-s $(m)$ circuit, then $L_{\mathrm{PSPACE}}^{m}$ has a size-s $(m)$ circuit.

Assuming Lemma 3.3 holds, we can finish the proof of Theorem 3.1, and show the desired lower bound on $L_{1}$. In particular, applying assumption (B) to Lemma 3.3 yields that $L_{\text {PSPACE }}^{s_{2}\left(n_{i}\right)}$ has a circuit of size $s\left(s_{2}\left(n_{i}\right)\right)$, for all sufficiently large $i$. By constraint (ii) $\left(s_{1}(n) \geq s\left(s_{2}(n)\right)\right), L_{\mathrm{PSPACE}}^{s_{2}\left(n_{i}\right)}$ has a circuit of size at most $s_{1}\left(n_{i}\right)$ for all sufficiently large $i$, so by definition of the $\ell$-function, $\ell\left(n_{i}\right) \geq s_{2}\left(n_{i}\right)$. But this implies $\alpha_{n_{i}}=s_{2}\left(n_{i}\right)$ for all sufficiently large $i$, which contradicts (3).

We now turn to showing how to prove Lemma 3.3. Intuitively, our strategy is to use $s(m)$-size circuits for $L_{1}^{m}$ to obtain $s(m)$-size circuits for $L_{\text {PSPACE }}$ on progressively larger and larger input lengths, using the downward self-reducibility of LPSPACE and the hypothesis that $L_{1}^{m}$ has small circuits.

Proof of Lemma 3.3. Let $m \in I$ be sufficiently large, and assume $L_{1}^{m}$ has a circuit $C_{m}$ of size $s(m)$. We want to prove that $L_{\mathrm{PSPACE}}^{m}$ has a circuit of size $s(m)$. Since $L_{\text {PSPACE }}^{1}$ has an $O(1)$-size size circuit (and $s(m)$ is increasing), it's clear that $L_{\text {PSPACE }}^{1}$ has a circuit of size $s(m)$ for all but finitely many input lengths $m$. We make the following inductive claim:

Amplification Claim: Under the hypothesis, if there is an $\ell<m$ such that $L_{\text {PSPACE }}^{\ell}$ has a size-s $(m)$ circuit, then $L_{\text {PSPACE }}^{\ell+1}$ also has a size- $s(m)$ circuit.

Assuming the claim with $\ell:=1$, we conclude that $L_{\mathrm{PSPACE}}^{\ell+1}=$ $L_{\text {PSPACE }}^{2}$ also has a circuit of size $s(m)$. Repeatedly applying the claim for $\ell=1$ up to $\ell=m-1$, we conclude that $L_{\mathrm{PSPACE}}^{m}$ has a circuit of size $s(m)$. That would complete the proof of the lemma.

To prove the Amplification Claim, suppose there is an $\ell<m$ such that $L_{\text {PSPACE }}^{\ell}$ has a size $s(m)$ circuit $D_{\ell}$. Using the downward selfreduction for $L_{\text {PSPACE}}$, we can also obtain a circuit $D_{\ell+1}^{\prime}$ for $L_{\text {PSPACE }}^{\ell+1}$, of size at most $O\left(s(m)^{2 d_{1}+1}\right)$. In particular, we can construct an $L_{\text {PSPACE }}^{\ell}$-oracle circuit for $L_{\text {PSPACE }}^{\ell+1}$ using the $O\left(n^{d_{1}}\right)$-time downward self-reduction. This oracle circuit has size at most $(\ell+1)^{2 d_{1}}$; if we replace each $L_{\text {PSPACE }}^{\ell}$ oracle query with the size- $s(m)$ circuit $D_{\ell}$, this yields a circuit $D_{\ell+1}^{\prime}$ of size at most $(\ell+1)^{2 d_{1}} \cdot s(m) \leq$ $s(m)^{2 d_{1}+1}$. By constraint (iii), we have that $D_{\ell+1}^{\prime}$ has size at most $s_{1}(m)$.

We want to use the assumed size-s $(m)$ circuit $C_{m}$ for $L_{1}^{m}$ to reduce the circuit complexity of $L_{\text {PSPACE }}^{\ell+1}$ down to $s(m)$. By (3), we have $\alpha_{m}<s_{2}(m)$ for sufficiently large $m \in I$, so (by the properties of the protocol $M_{1}$ ) the circuit $C_{m}$ implements $L_{\text {PSPACE }}$ for inputs of up to length $\alpha_{m}$.

We claim that by feeding the string $1^{m-\ell-1}$ to the first $m-\ell-1$ inputs of $C_{m}$, the remaining subcircuit of size at most $s(m)$ can compute $L_{\mathrm{PSPACE}}^{\ell+1}$ on all $x$ of length $\ell+1$. (Note that since we assume $\ell \leq m-1$, the padding amount $m-\ell-1 \geq 0$.) Since the circuit $D_{\ell+1}^{\prime}$ has size at most $s_{1}(m), L_{\text {PSPACE }}^{\ell+1}$ has a circuit of size at most $s_{1}(m)$. Therefore by the definition of $\alpha_{n}(2)$ and $\ell(n)$ (1), for our choice of $m$ we have $\alpha_{m} \geq \ell+1$.

Since $C_{m}$ implements $L_{\mathrm{PSPACE}}$ for inputs of up to length $\alpha_{m}$, and $\alpha_{m} \geq \ell+1$, the circuit

$$
C_{\ell+1}^{\prime}(x):=C_{m}\left(1^{m-\ell-1} x\right)
$$

is of size $s(m)$ and computes $L_{\mathrm{PSPACE}}^{\ell+1}$ correctly (by the paddability of $L_{\text {PSPACE}}$ ). This completes the proof of the Amplification Claim, and also the proof of Lemma 3.3.

This completes the proof of Theorem 3.1.

\section{SMALL CIRCUITS IMPLY SMALL WITNESSES}

In this section we prove our new Easy Witness Lemma, using the new Merlin-Arthur circuit lower bound (Theorem 3.1).

Lemma 4.1 (EAsy Witnesses For Low Nondeterministic Time). There are $e, g \geq 1$ such that for every increasing time-constructible $s(n), s_{2}(n):=s(e \cdot n)^{e}$, and $t(n)$,

if $\mathrm{NTIME}\left[O\left(t(n)^{e}\right)\right] \subset \operatorname{SIZE}[s(n)]$ then every $L \in \operatorname{NTIME}[t(n)]$ has witness circuits of size $s_{2}\left(s_{2}\left(s_{2}(n)\right)\right)^{2 g}$, provided that

(a) $s(n)<2^{n / e} / n$ and

(b) $t(n) \geq s_{2}\left(s_{2}\left(s_{2}(n)\right)\right)^{d}$ for a sufficiently large constant d.

For $s(n)=n^{k}$, if NP $\subset \operatorname{SIZE}\left[n^{k}\right]$, then NP has witness circuits of size $n^{O\left(k^{3}\right)}$ (Theorem 1.2).

For $s(n)=2^{(\log n)^{k}}$, NQP $\subset \operatorname{SIZE}\left[2^{(\log n)^{k}}\right]$ implies that NQP has witness circuits of size $2^{O\left((\log n)^{k^{3}}\right)}$ (Theorem 1.3).

Proof. Let $w(n):=s_{2}\left(s_{2}\left(s_{2}(n)\right)\right)^{2 g}$. Suppose

$$
\text { NTIME }\left[O\left(t(n)^{e}\right)\right] \subseteq \operatorname{SIZE}[s(n)],
$$

and assume NTIME $[t(n)]$ does not have $w(n)$-size witness circuits, i.e.,

there is some linear-time verifier $V$ such that

there are infinitely many "bad" inputs $x$, where

there is a $y_{x}$ of length $t(|x|)$ such that $V\left(x, y_{x}\right)$ accepts,

yet all such $y_{x}$ have circuit complexity at least $w(|x|)$.

We wish to establish a contradiction. Take $e \geq 1$ to be a sufficiently large parameter (all our constraints on $e$ will be constant lower 
bounds on it). Using our assumptions on $s(n)$ and $s_{2}(n)$, we claim that the hard MA language $L_{1}$ of Theorem 3.1 exists for

$$
s^{\prime}(n):=s(e \cdot n), s_{2}^{\prime}(n):=s^{\prime}(n)^{d^{\prime}} \text {, and } s_{1}^{\prime}(n):=s^{\prime}\left(s_{2}^{\prime}(n)\right),
$$

assuming $d^{\prime}$ is chosen such that $\max \left(2 d_{2}, 2 d_{1}+1, d_{3}+2\right) \leq d^{\prime} \leq e$

Let us check that these functions $s^{\prime}, s_{1}^{\prime}$, and $s_{2}^{\prime}$ satisfy all the constraints of Theorem 3.1:

- First, since $s(n)<2^{n / e} / n$ (by constraint (a)), we know that $s^{\prime}(n)=s(e \cdot n)<2^{e \cdot n / e} /(e \cdot n)<2^{n} /(2 n)$, for all sufficiently large $n$ and $e \geq 2$. Thus $s^{\prime}$ is a circuit-size function.

- For constraint (i), $d^{\prime} \geq 2 d_{2}$ implies that $s_{2}^{\prime}(n)=s^{\prime}(n)^{d^{\prime}} \geq$ $s^{\prime}(n)^{2 d_{2}}$.

- For constraint (ii), $d^{\prime} \geq 2 d_{1}+1$ implies $s_{1}^{\prime}(n)=s^{\prime}\left(s_{2}^{\prime}(n)\right) \geq s_{2}^{\prime}(n)=s^{\prime}(n)^{d^{\prime}} \geq s^{\prime}(n)^{2 d_{1}+1}$.

- Constraint (iii) $s_{1}^{\prime}(n) \geq s^{\prime}\left(s_{2}^{\prime}(n)\right)$ is trivially satisfied.

Thus by Theorem 3.1, $L_{1}$ is computable by an MA protocol in $O\left(s_{1}^{\prime}(n)^{2} \cdot s_{2}^{\prime}(n)^{d_{3}}\right)$ time with advice of length

$$
2 \log s_{2}^{\prime}(n)=2 d^{\prime} \log s^{\prime}(n)<2 d^{\prime} \cdot \log \left(2^{e \cdot n / e}\right)=2 d^{\prime} \cdot n .
$$

For $d^{\prime} \geq d_{3}+2$, we also have

$$
\begin{aligned}
s_{1}^{\prime}(n)^{2} \cdot s_{2}^{\prime}(n)^{d_{3}} & =s^{\prime}\left(s_{2}^{\prime}(n)\right)^{2} \cdot s_{2}^{\prime}(n)^{d_{3}} \leq s^{\prime}\left(s_{2}^{\prime}(n)\right)^{2+d_{3}} \\
& \leq s_{2}^{\prime}\left(s_{2}^{\prime}(n)\right) \leq s_{2}\left(s_{2}(n)\right)
\end{aligned}
$$

since $s_{2}^{\prime}(n)=s(e \cdot n)^{d^{\prime}} \leq s(e \cdot n)^{e}=s_{2}(n)$ for $d^{\prime} \leq e$. So $L_{1}$ is computable by an MA protocol in $O\left(s_{2}\left(s_{2}(n)\right)\right)$ time with $2 d^{\prime} \cdot n$ bits of advice, and has circuit lower bounds for size $s^{\prime}(n)=s(e \cdot n)$. That is, for all sufficiently large $n \in \mathbb{N}$, either

- the circuit complexity of $L_{1}^{n}$ is greater than $s(e \cdot n)$, or

- the circuit complexity of $L_{1}^{s_{2}^{\prime}(n)}$ is greater than $s\left(e \cdot s_{2}^{\prime}(n)\right)$.

By (5), there are infinitely many input lengths $\left\{n_{i}\right\}$ such that, by encoding a bad input $x_{i}$ of length $n_{i}$ as advice and guessing a valid $y_{i}$ such that $V\left(x_{i}, y_{i}\right)$ accepts, we can nondeterministically guess and verify the truth table of a function $y_{i}$ with circuit complexity at least $w\left(\left|x_{i}\right|\right)$.

Our next step is to use these bad $x_{i}$ 's to derandomize the MA protocol defining $L_{1}$ : we give a nondeterministic algorithm $N$ such that for all $i$ and all inputs of length $n_{i}, N$ uses $\left(2 d^{\prime}+1\right) n_{i}$ bits of advice, runs in $O\left(t\left(n_{i}\right)^{e}\right)$ time, and correctly decides $L_{1}$ on all $n_{i}$-bit inputs. On inputs of length $n_{i}$, the advice to $N$ is:

- a bad input $x_{\text {hard }}$ of length $n_{i}$, and

- the advice $\alpha_{n_{i}}$ used by the Merlin-Arthur protocol $M_{1}$ of Theorem 3.1, on inputs of length $n_{i}$.

On inputs of length $s_{2}^{\prime}\left(n_{i}\right)$, the advice to $N$ is $x_{\text {hard }}$ as well as the advice $\alpha_{s_{2}^{\prime}\left(n_{i}\right)}$ used by $M_{1}$.

On inputs $x$ of length $n_{i}$ and inputs $x$ of length $s_{2}^{\prime}\left(n_{i}\right), N$ first guesses a string $y_{\text {hard }}$ of length $O\left(t\left(n_{i}\right)\right)$ and runs $V\left(x_{\text {hard }}, y_{\text {hard }}\right)$. If $V$ rejects, then $N$ rejects. Otherwise, $N$ will use $y_{\text {hard }}$ as a hard function in the pseudorandom generator $G(\cdot, \cdot)$ of Theorem 2.2, for fooling circuits of size $w(n)^{1 / g}=s_{2}\left(s_{2}\left(s_{2}(n)\right)\right)^{2}$.

Fix a constant $a \geq 1$ such that $G\left(y_{\text {hard }}, \cdot\right)$ runs in $O\left(\left|y_{\text {hard }}\right|^{a}\right)$ time. Let $\ell_{i} \in\left\{n_{i}, s_{2}^{\prime}\left(n_{i}\right)\right\}$, depending on the current input length. Our $N$ constructs a circuit $C_{x}(\cdot, \cdot)$ simulating the deterministic $O\left(s_{2}\left(s_{2}\left(\ell_{i}\right)\right)\right)$-time part of $M_{1}(x)$. In particular, the circuit $C_{x}$ takes a "nondeterministic" input $z$ of length at most $s_{2}\left(s_{2}\left(\ell_{i}\right)\right)^{2}$, as well as "randomness" input $r$ of length at most $s_{2}\left(s_{2}\left(\ell_{i}\right)\right)^{2}$, and $C$ has size at most $s_{2}\left(s_{2}\left(\ell_{i}\right)\right)^{2} \leq s_{2}\left(s_{2}\left(s_{2}\left(n_{i}\right)\right)\right)^{2}$. Then $N$ nondeterministically guesses an input $z$, and enumerates all $s=\left|y_{\text {hard }}\right|^{g}$ seeds to the generator $G\left(y_{\text {hard }}, \cdot\right)$, obtaining output strings $r_{1}, \ldots, r_{s}$ in $O\left(\left|y_{\text {hard }}\right|^{a+g}\right)=O\left(t\left(n_{i}\right)^{a+g}\right)$ time. (We emphasize that this is done for both $\ell_{i}=n_{i}$ and $\ell_{i}=s_{2}^{\prime}\left(n_{i}\right)$.) Finally, $N$ computes the probability that $C_{x}\left(y_{\text {hard }}, r_{j}\right)$ accepts over all $j=1, \ldots, s$. If this probability is greater than $1 / 2$, then $N$ accepts else it rejects.

In total, $N$ takes time

$$
\begin{aligned}
O\left(s_{2}\left(s_{2}\left(n_{i}\right)\right)^{2}+\right. & \left.t\left(n_{i}\right)^{a+g}+t\left(n_{i}\right)^{g} \cdot s_{2}\left(s_{2}\left(n_{i}\right)\right)^{2}\right) \\
= & O\left(t\left(n_{i}\right)^{a+g}\right)
\end{aligned}
$$

on inputs of length $n_{i}$. Note the $y_{\text {hard }}$ for an input $x_{\text {hard }}$ of length $n_{i}$ has circuit complexity at least $s_{2}\left(s_{2}\left(s_{2}\left(n_{i}\right)\right)\right)^{2 g}$. Since the circuit $C_{x}(\cdot, \cdot)$ for inputs $x$ of length $\ell_{i}=s_{2}^{\prime}\left(n_{i}\right)$ has size $s_{2}\left(s_{2}\left(s_{2}^{\prime}\left(n_{i}\right)\right)\right)^{2} \leq$ $s_{2}\left(s_{2}\left(s_{2}\left(n_{i}\right)\right)\right)^{2}$, the same advice $x_{\text {hard }}$ can also be used with the generator $G(\cdot, \cdot)$ to derandomize $M_{1}$ on inputs of length $s_{2}^{\prime}\left(n_{i}\right)$ as well. Thus on inputs of length $s_{2}^{\prime}\left(n_{i}\right), N$ takes $n_{i}+2 d^{\prime} \cdot s_{2}^{\prime}\left(n_{i}\right)$ advice and runs in time

$$
\begin{gathered}
O\left(s_{2}\left(s_{2}\left(s_{2}\left(n_{i}\right)\right)\right)^{2}+t\left(n_{i}\right)^{a+g}+t\left(n_{i}\right)^{g} \cdot s_{2}\left(s_{2}\left(s_{2}\left(n_{i}\right)\right)\right)^{2}\right) \\
\leq O\left(t\left(n_{i}\right)^{a+g}\right) \leq O\left(t\left(s_{2}^{\prime}\left(n_{i}\right)\right)^{a+g}\right)
\end{gathered}
$$

where in the next-to-last inequality, we have applied constraint (b).

If we set $e \geq a+g$, then by (4), the nondeterministic $O\left(t(n)^{e}\right)$ time algorithm $N$ (using $\left(2 d^{\prime}+1\right) n$ bits of advice) described in the previous paragraph has circuits of size $s\left(\left(2 d^{\prime}+2\right) n\right)$. In particular, for almost every input length in the set $\left\{n_{i}\right\}$, the language $L_{1}$ has circuits of size $s\left(\left(2 d^{\prime}+2\right) n_{i}\right)$ on $n_{i}$-bit inputs, and has circuits of size $s\left(\left(2 d^{\prime}+1\right) \cdot s_{2}^{\prime}\left(n_{i}\right)+n_{i}\right) \leq s\left(\left(2 d^{\prime}+2\right) \cdot s_{2}^{\prime}\left(n_{i}\right)\right)$ on $s_{2}^{\prime}\left(n_{i}\right)$-bit inputs.

For $e \geq 2 d^{\prime}+2$, this contradicts the circuit lower bound of Theorem 3.1.

\section{APPLICATIONS}

Now we turn to showing how the new Easy Witness Lemmas imply stronger algorithm-to-lower bound connections. Recall a nondeterministic algorithm for GAP $C$ UnSAT outputs yes, no, or don't know on every computation path, it outputs no on some computation path if its input circuit has at least $1 / 4$ of its assignments satisfying, outputs yes on some path if its input circuit is unsatisfiable, and for every input circuit, the algorithm never outputs yes on some path and no on another path.

Reminder of Theorem 1.4 Let $C$ be typical, and let $\varepsilon \in(0,1)$. Suppose GAP $C$ UNSAT on n-input, $2^{\varepsilon n}$-size circuits is solvable in nondeterministic $O\left(2^{(1-\varepsilon) n}\right)$ time. Then there is a $c \geq 1$ such that for all $k$, NTIME $\left[n^{c k^{4} / \varepsilon}\right]$ does not have $n^{k}$-size $C$-circuits.

Proof. The proof is similar to earlier arguments [21, 25, 27]. Let $\varepsilon \in(0,1)$ be given. Assume

(A) for all $c \geq 1$, there is a $k \geq 1$ such that NTIME $\left[n^{c k^{4} / \varepsilon}\right]$ has $n^{k}$-size $C$-circuits, and

(B) GaP $C$ Unsat on $n$-input $2^{\varepsilon n}$-size circuits is in $O\left(2^{(1-\varepsilon) n}\right)$ time. 
Let $c$ be sufficiently large in the following, and let

$$
t(n)=n^{c k^{4} / \varepsilon}
$$

for notational convenience. Since $C$ is evaluatable (given a circuit from the class, we can evaluate it on an input in polynomial time), from (A) it follows that NTIME $[t(n)]$ has $n^{O(k)}$-size unrestricted circuits (e.g., over the basis AND/OR/NOT of fan-in two). By the Easy Witness Lemma for NP (Lemma 1.2), NTIME $[t(n)]$ has $n^{O\left(k^{3}\right)}$-size witness circuits for every NP verifier. We choose the PCP verifier of Ben-Sasson and Viola [4], which yields proofs which are quasilinear for any running time $T(n)$. In particular, for every verifier algorithm $V(x, y)$ running in time $T(n) \geq n$, they give an algorithm $A$ running in poly $(n, \log T(n))$ time which, given $x \in\{0,1\}^{n}$, outputs an oracle circuit $C_{x}^{O}$ with the following properties: ${ }^{4}$

- $C_{x}^{O}$ has $\ell=\log _{2}(T(n))+O(\log \log T(n))$ inputs, poly $(n, \log T(n))$ size, and each copy of the oracle gate $O$ in $C_{x}^{O}$ has $\ell$ inputs as well.

- If there is a $y$ of length $T(n)$ such that $V(x, y)$ accepts, then there is an oracle $O:\{0,1\}^{\ell} \rightarrow\{0,1\}$ such that $C_{x}^{O}$ is unsatisfiable.

- If $V(x, y)$ rejects on all $y$ of length $T(n)$, then for every oracle $O:\{0,1\}^{\ell} \rightarrow\{0,1\}, C_{x}^{O}$ has at least $2^{\ell} \cdot(1-1 / n)$ satisfying assignments.

Note the oracle $O$ acts as a witness for the PCP verifier which runs the above transformation on $x$, and checks whether $C_{x}^{O}$ is unsatisfiable or not by guessing random inputs to $C_{x}^{O}$.

Take a language $L \in \operatorname{NTIME}[t(n)]-\operatorname{NTIME}\left[t(n)^{1-\varepsilon / 2}\right]$ such that $L \subseteq\left\{1^{n} \mid n \geq 0\right\}[22,30]$, and let $V$ be a $O(t(n))$-time verifier for $L$. On an input $1^{n}$, let $N$ be a nondeterministic algorithm which:

(1) runs the algorithm $A$ in poly $(n, \log t(n))$ time to produce a circuit $C_{n}^{O}$,

(2) guesses a witness circuit $W_{n}$ of size $n^{O\left(k^{3}\right)}$ encoding the oracle $O$ for the PCP verifier,

(3) plugs the circuit $W_{n}$ in place of the oracle $O$ in the circuit $C_{n}^{O}$, obtaining a circuit of size $n^{O\left(k^{3}\right)} \cdot \operatorname{poly}(n, \log t(n)) \leq n^{O\left(k^{3}\right)}$,

(4) checks satisfiability of $C_{n}^{W_{n}}$ by exhaustive search, in time $2^{\ell} \cdot n^{O\left(k^{3}\right)} \leq t(n) \cdot$ poly $(\log t(n)) \cdot n^{O\left(k^{3}\right)} \leq n^{c k^{4} / \varepsilon+O\left(k^{3}\right)}$.

Under the hypotheses, we will show how to simulate $N$ in nondeterministic time $t(n)^{1-\varepsilon} \cdot$ poly $(\log t(n))$, implying a contradiction. If we had an unrestricted CircuIT SAT algorithm or GAP CIRCUIT UNSAT algorithm running in $O\left(2^{\ell(1-\varepsilon)}\right) \leq t(n)^{1-\varepsilon+o(1)}$ time, this would be easy: we could just use the nondeterministic GAP CIRCUIT UNSAT algorithm to speed up item 4 in the description above. Because we only have a GAP $C$ UnSAT algorithm, and $C$ might be weaker than unrestricted circuits (as far as we know), the argument becomes more complicated.

Consider the task:

EvAl-Gate: Given a circuit $C^{O}$ of $n$ size, a circuit of size at most $n$ encoding an oracle $O$, an input $x$ of length $\ell$, and an integer $i=1, \ldots, n$, what is the output of the $i$-th gate of $C^{O}$ evaluated on $x$ ?

Clearly EvAL-GATE is computable in polynomial time. By assumption (A), Eval-Gate has $C$-circuits of size $n^{O(k)}$. In lieu of item

\footnotetext{
${ }^{4}$ This is not formally stated in their article, but it is immediate from their Theorem 1.1
}

4 in the description of $N$, our new nondeterministic algorithm $N^{\prime}$ guesses a $C$-circuit $E$ computing Eval-Gate which can take $C_{n}^{O}$ and $W_{n}$ as inputs; since both have size $n^{O\left(k^{3}\right)}$, a $C$-circuit of $n^{O\left(k^{4}\right)}$ suffices. For every $i=1, \ldots, s$ of $C_{n}^{W_{n}}$ (where $\left.s=n^{O\left(k^{3}\right)}\right)$, $E\left(C_{n}^{O}, W_{n}, x, i\right)$ outputs the value of the $i$-th gate of $C_{n}^{W_{n}}(x)$; by convention, we let the $s$-th gate be the output of the circuit. Assuming $E$ is a circuit correctly computing EvAL-GATE on the appropriate input length, it is easy to see that

$$
E\left(C_{n}^{O}, W_{n}, x, s\right)=1 \Longleftrightarrow C_{n}^{W_{n}}(x)=1 .
$$

Without loss of generality, assume all gates of $C_{n}^{W_{n}}$ have a fixed gate type (e.g., NAND). For each gate $i$ of $C_{n}^{W_{n}}$, let $i_{1}, i_{2}<i$ be the indices of the two gates of $C_{n}^{W_{n}}$ whose outputs are the two inputs to gate $i$. Let $F_{i}\left(g_{i}, g_{i_{1}}, g_{i_{2}}\right)$ be the canonical 3-CNF on three variables (where $g_{j}$ corresponds to the output of gate $j$ ) which is true if and only if the outputs of gates $i_{1}$ and $i_{2}$ are consistent with the output of gate $i$. Now consider the circuit $D(x)$, which outputs the NAND over all $i=1, \ldots, s$ of

$$
F_{i}\left(E\left(C_{n}^{O}, W_{n}, x, g_{i}\right), E\left(C_{n}^{O}, W_{n}, x, g_{i_{1}}\right), E\left(C_{n}^{O}, W_{n}, x, g_{i_{2}}\right)\right) .
$$

It is easy to see that $D(x)=0$ if and only if $E$ is consistent for all gates of $C_{n}^{W_{n}}$ on the input $x$; that is, $E$ claims correct outputs for every gate of the circuit $C_{n}^{W_{n}}$ on the input $x$. From this, we have the consistency condition:

$$
\text { for all } x, D(x)=0 \text { implies that } E\left(C_{n}^{O}, W_{n}, x, s\right)=C_{n}^{W_{n}}(x) \text {. }
$$

Furthermore, since $C$ is closed under conjunctions and projections, given $E, C_{n}^{O}$, and $W_{n}$, the circuit $D$ can be computed in time $n^{O\left(k^{4}\right)}$, where $D$ is a circuit from $C$. By our choice of $t(n)$ (with sufficiently large $c$ ),

$$
n^{O\left(k^{4}\right)} \leq 2^{\varepsilon \ell} \leq t(n)^{\varepsilon} \cdot \text { poly }(\log t(n)) .
$$

So the circuit $D$ has $\ell$ inputs and size $2^{\varepsilon \ell}$, so our nondeterministic algorithm $N^{\prime}$ can run the nondeterministic GAP $C$ UNSAT algorithm on $D$, assumed by (B). $N^{\prime}$ rejects if the nondeterministic GAPUNSAT algorithm outputs no or don't know on D. Otherwise, $N^{\prime}$ accepts if and only if the nondeterministic GAP-UNSAT algorithm outputs yes on $E\left(C_{n}^{O}, W_{n}, \cdot, s\right)$ (with $\ell$ free inputs).

The running time of $N^{\prime}$ is

$$
n^{O\left(k^{4}\right)}+2^{(1-\varepsilon) \ell} \leq t(n)^{1-\varepsilon} \cdot \operatorname{poly}(\log t(n)) .
$$

By the properties of the PCP verifier and our GAP-UNSAT algorithm, we have:

- If $1^{n} \in L$, then there is a $W_{n}$ and an $E$ such that $D$ is unsatisifiable. Then, $N^{\prime}$ accepts $1^{n}$ on the correct guesses of $W_{n}$ and $E$. In particular, the GAP-UNSAT algorithm will report yes on $D$ (since $D$ is unsatisfiable) on some path, and report yes on $E\left(C_{n}^{O}, W_{n}, \cdot, s\right)$ on some path as well.

- If $1^{n} \notin L$, for all choices of $W_{n}$, the circuit $C_{n}^{W_{n}}$ has at least $2^{\ell} \cdot(1-1 / n)$ satisfying assignments. The algorithm $N^{\prime}$ guesses a circuit $E$ for EvAL-GATE and constructs the circuit $D$. If our GAP-UNSAT algorithm returns no or don't know on $D$, then $N^{\prime}$ rejects by definition. If GAP-UNSAT returns yes 
on some path, then it must be that $D$ has less than $2^{\ell} / 4$ satisfying assignments (if it had more, the GAP-UNSAT algorithm would return no on some path, and therefore never output yes on any path, by definition). So $D(x)=0$ on at least $3 / 4$ of the inputs $x$. By (6), $E\left(C_{n}^{O}, W_{n}, x, s\right)=C_{n}^{W_{n}}(x)$ for at least $3 / 4$ of the inputs $x$. Since $C_{n}^{W_{n}}$ has at least $2^{\ell} \cdot(1-1 / n)$ satisfying assignments, it must be that the circuit $E\left(C_{n}^{O}, W_{n}, \cdot, s\right)$ of $\ell$ inputs has at least $2^{\ell} \cdot(1-1 / n-1 / 4) \geq 2^{\ell} / 4$ satisfying assignments. Therefore the GAP-UNSAT algorithm on the circuit $E\left(C_{n}^{O}, W_{n}, \cdot, s\right)$ will not return yes on any computation path (because it must return no on some path, and it never outputs contradictory answers on the same circuit). Thus $N^{\prime}$ rejects $1^{n}$ on all computation paths.

In summary, $N^{\prime}$ correctly decides the language $L$. This is a contradiction, as $N^{\prime}$ runs in $t(n)^{1-\varepsilon} \cdot$ poly $(\log t(n))$ time.

REMARK 1. Fortnow and Santhanam [9] proved that for all polynomials $t(n)$, there is an $\alpha>0$ and a language

$L \in \mathrm{NTIME}[t(n)]$ that is not in NTIME $\left[t(n)^{1-\varepsilon / 2}\right] / n^{\alpha}$. If we use this $L$ in the above proof, then the nondeterministic GAP $C$ SAT algorithm could even use $2^{\beta n}$ bits of advice on $n$-input circuits of size $2^{\varepsilon n}$ (for small enough $\beta>0$ depending on $\varepsilon$ and $k$ ), and still yield the desired lower bound.

Reminder of Theorem 1.5 Let $C$ be typical, and let $\varepsilon \in(0,1)$. Suppose GAP $C$ UNSAT on n-input, $2^{n^{\varepsilon}}$-size circuits is in nondeterministic $O\left(2^{n-n^{\varepsilon}}\right)$ time. Then for all $k$, there is a $c \geq 1$ such that NTIME $\left[2^{\log ^{c k^{4} / \varepsilon} n}\right]$ does not have $2^{\log ^{k} n}$-size $C$-circuits.

Proof. We use the exact same strategy as Theorem 1.4, with the following minor modifications. Assume

(A) for all $c$, there is a $k \geq 1$ such that NTIME $\left[2^{\log ^{c k^{4} / \varepsilon} n}\right]$ has $2^{\log ^{k} n}$-size $C$-circuits, and

(B) GAP $C$ UnSAT on $n$-input $2^{n^{\varepsilon}}$-size circuits is in $O\left(2^{n-n^{\varepsilon}}\right)$ time.

Set $t(n)=2^{\log ^{c k^{4} / \varepsilon} n}$. As argued in Theorem 1.4, by the Easy Witness Lemma for NQP (Lemma 1.2), NTIME $[t(n)]$ has $2^{O\left(\log ^{k} n\right)}$. size witness circuits for every NP verifier. Use the PCP verifier of Ben-Sasson and Viola [4] as before which outputs a circuit $C_{n}^{O}$ of poly $(n)$ size with number of inputs

$$
\ell=\left(\log _{2} n\right)^{c k^{4} / \varepsilon}+O(\log \log n) .
$$

Take $L \in \operatorname{NTIME}[t(n)]-\operatorname{NTIME}\left[t(n) / 2^{\log ^{\varepsilon / 2} t(n)}\right]$ such that $L \subseteq$ $\left\{1^{n} \mid n \geq 0\right\}[22,30]$, and let $V$ be a $O(t(n))$-time verifier for $L$. On an input $1^{n}$, let $N$ be a nondeterministic algorithm which:

(1) runs in poly $(n)$ time to produce a circuit $C_{n}^{O}$,

(2) guesses a witness circuit $W_{n}$ of size $2^{O\left(\log ^{k^{3}} n\right)}$ encoding the oracle $O$ for the PCP verifier,

(3) plugs the circuit $W_{n}$ in place of the oracle $O$ in the circuit $C_{n}^{O}$, obtaining a circuit of size $2^{O\left(\log ^{k^{3}} n\right)} \cdot$ poly $(n) \leq 2^{O\left(\log ^{k^{3}} n\right)}$,
(4) checks satisfiability of $C_{n}^{W_{n}}$ by exhaustive search, in time $2^{\ell} \cdot 2^{O\left(\log ^{3} n\right)} \leq 2^{\log ^{c} k^{4} / \varepsilon} n+O\left(\log ^{k^{3}} n\right)$.

As before, we show how to simulate $N$ faster. We define the EvalGATE problem as before; by assumption (A), it has $\mathcal{C}$-circuits of size $2^{\log ^{k} n}$. Thus there is a circuit $E$ of size $2^{O\left(\log ^{k^{4}} n\right)}$ which can take $W_{n}$ and $C_{n}^{O}$ as input and faithfully simulate EvAL-GATE. Also as before, we construct the circuit $D$ of size $2^{O\left(\log ^{4} n\right)}$ and of $\ell$ inputs. For sufficiently large $c$, the size of $D$ is

$$
2^{O\left(\log ^{k^{4}} n\right)} \leq 2^{\log ^{\varepsilon} t(n)} \leq 2^{\left(\left(\log ^{c k^{4}} n\right)+O(\log \log n)\right)^{\varepsilon}} \leq 2^{\ell^{\varepsilon}},
$$

so the assumed nondeterministic GAP $C$ UnSAT algorithm can be run on $D$, and it can be run on the circuit $E\left(C_{n}^{O}, W_{n}, \cdot, g_{i}\right)$, obtaining yes/no answers in nondeterministic time

$$
O\left(2^{\ell-\ell^{\varepsilon}}\right) \leq t(n) / 2^{\log ^{\varepsilon} t(n)} .
$$

Following the rest of the proof of Theorem 1.4, we can decide $L$ in nondeterministic time $t(n) / 2^{\log ^{\varepsilon} t(n)}$, which is a contradiction. $\quad \square$

Finally, to conclude lower bounds such as NQP $\not \subset$ ACC $\circ$ THR, we only have to appeal to the ACC $\circ$ THR-SAT algorithm:

Theorem 5.1 ([26]). For all integers $d$ and $m \geq 2$, there is an $\varepsilon>0$ and an $O\left(2^{n-n^{\varepsilon}}\right)$-time deterministic algorithm for the satisfiability problem on depth-d size- $2^{n^{\varepsilon}}$ circuits over unbounded fan-in AND, OR, and MODm gates, with linear threshold gates at the bottom layer.

The following is immediate:

Reminder of Theorem 1.6 For every $c, d$, and $m \geq 2$, there is an $e \geq 1$ and a problem in NTIME $\left[n^{\log e} n\right]$ which does not have depth-d $n^{\log ^{c} n}$-size AC $[m]$ circuits, even with $n^{\log ^{c} n}$ linear threshold gates at the bottom layer.

Note the lower bound exponent $e$ of Theorem 1.6 does, in principle, depend on the depth of the circuit and the modulus $m$ of the MOD gates. By a standard trick, it is easy to remove this dependence:

Corollary 5.2. There is a e $\geq 1$ such that NTIME $\left[n^{\log ^{e} n}\right]$ does not have polynomial-size ACC ○ THR circuits.

Proof. Assume for a contradiction that for all $e$ there is a $d$ such that NTIME $\left[n^{\log ^{e} n}\right]$ has depth- $d$ ACC $\circ$ THR circuits of size $O\left(n^{d}\right)$. We observe the quantifiers can be rearranged somewhat, in that the assumption implies:

$\left(^{*}\right)$ There are universal constants $d_{0}, m_{0} \geq 1$ such that for all $e$, there is a $d$ such that NTIME $\left[n^{\log ^{e} n}\right]$ has depth- $d_{0}$ AC $\left[m_{0}\right] \circ$ THR circuits of size $O\left(n^{d}\right)$.

Statement $\left({ }^{*}\right)$ is true because assuming $\mathrm{P}$ is in ACC $\circ$ THR of polynomial size, Circuit Evaluation has ACC $\circ$ THR circuits of polynomial size. As a consequence, there are $d_{0}, m_{0}$ such that any circuit of size $s$ (of any depth) can be simulated by an AC $\left[m_{0}\right] \circ \mathrm{THR}$ circuit of $s^{d_{0}}$ size and depth $d_{0}$. However, ( $\left.{ }^{*}\right)$ directly contradicts Theorem 1.6 with $c=1$. 


\section{CONCLUSION}

We have shown how slightly faster CIRCUIT SAT algorithms, even those distinguishing unsatisifiable circuits from circuits with many satisfying assigments, can also imply circuit lower bounds for the nondeterministic classes NP and NQP. This suggests that the ageold problem of proving that NP does not have linear-size circuits may well be in striking distance, or at least that we may find nonlinear lower bounds for weak circuit classes in the near future (however, note $\mathrm{E}^{\mathrm{NP}} \not \subset$ SIZE $[O(n)]$ is still open, and one has to prove this first!). We would like to draw attention to a few related open problems:

NP versus ACC? The main obstacle to proving NP $\not \subset A C C$ is the lack of a GAP ACC UNSAT algorithm which runs in $2^{(1-\varepsilon) n}$ time on $2^{\varepsilon n}$-size circuits, for some $\varepsilon>0$. The ACC-SAT algorithm used in the NQP lower bound applies the well-known transformation of ACC to SYM $\circ$ AND [3, 29], which blows up the circuit by a quasi-polynomial factor; because of this blow-up it does not seem possible to get a much faster GAP ACC UNSAT algorithm using this transformation. But perhaps a better SAT algorithm can be derived under the assumption NP $\subset$ ACC.

EXP or BPEXP lower bounds? We now have interesting circuit lower bounds for Nondeterministic Quasi-Polynomial Time (NQP). We do not believe that NQP $\subseteq$ EXP, but it seems very unlikely that EXP-complete problems are solvable in NQP; EXPcomplete problems ought to be just as hard. Can the framework be extended further to show that faster CIRCUIT SAT or GAP UNSAT algorithms imply EXP lower bounds? How about lower bounds for the randomized exponential time class, BPEXP? It is known that if we have non-trivial learning algorithms for circuit classes with appropriate parameters, then BPEXP lower bounds follow $[8,19]$. Perhaps SAT algorithms can help expedite learning?

Reduce $\mathbf{E}^{\mathrm{NP}}$ ? In several settings $[5,13,25]$, it is known that faster SAT algorithms for weak classes of formulas would imply a circuit lower bound for the (huge) class $\mathrm{E}^{\mathrm{NP}}$. For example, a $1.9^{n}$ time nondeterministic CNF-UNSAT algorithm would imply $\mathrm{E}^{\mathrm{NP}}$ does not have linear-size "Valiant-series-parallel" circuits $[5,13]$. When can the gigantic class $\mathrm{E}^{\mathrm{NP}}$ be reduced to a smaller class?

\section{ACKNOWLEDGMENTS}

We thank Marco Carmosino, Valentine Kabanets, Igor Carboni Oliveira, and the STOC reviewers for helpful comments.

\section{REFERENCES}

[1] Sanjeev Arora and Boaz Barak. 2009. Computational Complexity - A Modern Approach. Cambridge University Press.

[2] László Babai, Lance Fortnow, Noam Nisan, and Avi Wigderson. 1993. BPP Has Subexponential Time Simulations Unless EXPTIME has Publishable Proofs. Computational Complexity 3, 4 (1993), 307-318.
[3] Richard Beigel and Jun Tarui. 1994. On ACC. Computational Complexity (1994), 350-366.

[4] Eli Ben-Sasson and Emanuele Viola. 2014. Short PCPs with projection queries. In ICALP. 163-173.

[5] Marco L. Carmosino, Jiawei Gao, Russell Impagliazzo, Ivan Mihajlin, Ramamohan Paturi, and Stefan Schneider. 2016. Nondeterministic Extensions of the Strong Exponential Time Hypothesis and Consequences for Non-reducibility. In Proceedings of the ACM Conference on Innovations in Theoretical Computer Science (ITCS). 261-270.

[6] Shiteng Chen and Periklis A. Papakonstantinou. 2016. Depth-Reduction for Composites. In FOCS. 99-108.

[7] Ning Ding. 2015. Some New Consequences of the Hypothesis That P Has Fixed Polynomial-Size Circuits. In International Conference on Theory and Applications of Models of Computation (TAMC). Springer, 75-86.

[8] Lance Fortnow and Adam R. Klivans. 2009. Efficient learning algorithms yield circuit lower bounds. 7. Comput. System Sci. 75, 1 (2009).

[9] Lance Fortnow and Rahul Santhanam. 2016. New Non-Uniform Lower Bounds for Uniform Classes. In CCC. 19:1-19:14.

[10] Lance Fortnow and Rahul Santhanam. 2017. Robust simulations and significant separations. Inf. Comput. 256 (2017), 149-159.

[11] Lance Fortnow, Rahul Santhanam, and Ryan Williams. 2009. Fixed-polynomial size circuit bounds. In CCC. IEEE, 19-26.

[12] Russell Impagliazzo, Valentine Kabanets, and Avi Wigderson. 2002. In search of an easy witness: Exponential time vs. probabilistic polynomial time. 7. Comput. System Sci. 65, 4 (2002), 672-694.

[13] Hamid Jahanjou, Eric Miles, and Emanuele Viola. 2015. Local reductions. In ICALP. Springer, 749-760.

[14] Ravi Kannan. 1982. Circuit-Size Lower Bounds and Non-Reducibility to Sparse Sets. Information and Control 55, 1 (1982), 40-56.

[15] Richard Karp and Richard Lipton. 1982. Turing Machines That Take Advice. L'Enseignement Mathématique 28, 2 (1982), 191-209.

[16] Johannes Kobler and Osamu Watanabe. 1998. New Collapse Consequences of NP Having Small Circuits. SIAM 7. Comput. 28, 1 (1998), 311-324.

[17] Richard Lipton. 1994. Some Consequences of Our Failure to Prove Non-Linear Lower Bounds on Explicit Functions. In Structure in Complexity Theory. 79-87.

[18] Peter Bro Miltersen, N. V. Vinodchandran, and Osamu Watanabe. 1999. Superpolynomial versus half-exponential circuit size in the exponential hierarchy. In COCOON (LNCS 1627). Springer, 210-220.

[19] Igor Carboni Oliveira and Rahul Santhanam. 2017. Conspiracies Between Learning Algorithms, Circuit Lower Bounds, and Pseudorandomness. In CCC. 18:118:49.

[20] Rahul Santhanam. 2009. Preliminary version in STOC'07. Circuit Lower Bounds for Merlin-Arthur Classes. SIAM f. Comput. 39, 3 (2009. Preliminary version in STOC'07), 1038-1061.

[21] Rahul Santhanam and Ryan Williams. 2013. Preliminary version in CCC'13.. On Uniformity and Circuit Lower Bounds. Computational Complexity 23, 2 (2013. Preliminary version in CCC'13.), 177-205.

[22] Joel Seiferas, Michael Fischer, and Albert Meyer. 1978. Separating Nondeterministic Time Complexity Classes. JACM 25, 1 (1978), 146-167.

[23] Luca Trevisan and Salil P. Vadhan. 2007. Preliminary version in CCC'02. Pseudorandomness and Average-Case Complexity Via Uniform Reductions. Computational Complexity 16, 4 (2007. Preliminary version in CCC'02), 331-364.

[24] Christopher Umans. 2003. Pseudo-random Generators for All Hardnesses. $\mathcal{F}$. Comput. System Sci. 67, 2 (2003), 419-440.

[25] Ryan Williams. 2013. See also STOC'10.. Improving exhaustive search implies superpolynomial lower bounds. SIAM f. Comput. 42, 3 (2013. See also STOC'10.), $1218-1244$

[26] Ryan Williams. 2014. New algorithms and lower bounds for circuits with linear threshold gates. In STOC. 194-202.

[27] Ryan Williams. 2014. See also CCC'11. Nonuniform ACC Circuit Lower Bounds. FACM 61, 1 (2014. See also CCC'11), 2.

[28] R. Ryan Williams. 2016. Natural Proofs versus Derandomization. SIAM f. Comput. 45, 2 (2016), 497-529.

[29] Andrew Chi-Chih Yao. 1990. On ACC and threshold circuits. In FOCS. 619-627.

[30] Stanislav Žák. 1983. A Turing machine time hierarchy. Theoretical Computer Science 26, 3 (1983), 327-333. 